\title{
Molecular recognition through divalent interactions with a self-assembled fibrillar network of a supramolecular organogel
}

\author{
Beatriu Escuder,* Juan F. Miravet* and José A. Sáez \\ Received 21st July 2008, Accepted 16th September 2008 \\ First published as an Advance Article on the web 20th October 2008 \\ DOI: 10.1039/b812520a
}

The interaction of phenol derivatives with the self-assembled fibrillar network of two different supramolecular gels has been studied. NMR relaxometry reveals the selective interaction of resorcinol over other related molecules with a gel formed by the gelator $\mathbf{2}$ which contains terminal pyridine units. No selectivity is observed for a related gelator that contains phenyl instead of pyridine moieties. The selectivity observed by NMR experiments permits the selective suppression of the ${ }^{1} \mathrm{H}$ NMR signals of resorcinol. This behaviour is translated to macroscopic properties such as the thermal stability of the gels. The observed selectivity together with X-ray diffraction data and molecular modelling suggest that the gels formed by $\mathbf{2}$ present arrays of pyridine H-bond acceptor groups capable of selective multivalent interaction with phenolic substrates.

\section{Introduction}

Molecular recognition of a substrate by a host molecule is a main goal in the work related to supramolecular chemistry. It has been described extensively that molecular recognition in solution can be achieved with the use of host molecules containing the appropriate arrangement of binding groups. ${ }^{1}$ Often, the preparation of the receptor molecules requires considerable synthetic effort in order to fulfil the required structural characteristics. A very convenient alternative from the point of view of synthetic economy is the use of self-assembled host systems. Relevant examples of molecular recognition by non-covalently assembled systems have been reported, for example, with the use of rosettes, ${ }^{2}$ capsules $^{3}$ and nanoreactors. ${ }^{4}$ Related to this, the use of self assembled monolayers as multivalent hosts that would permit the selective positioning or immobilization of (bio)molecules on surfaces has been described. ${ }^{5}$ Additionally, there is an increasing interest in the potential of multivalent interactions in supramolecular chemistry. ${ }^{6}$

Following the growing activity in the study of functional supramolecular gels, ${ }^{7}$ which has produced new materials with exciting properties in recent years, we are interested in the use of this type of soft matter in molecular recognition. Supramolecular gels are formed by anisotropic aggregation of low molecular weight molecules This process results in the formation of self-assembled fibrillar networks that percolate the solvent and produce gelation. In many cases the fibrillar structures are microcrystalline, indicating that the fibres consist of a well ordered array of molecules. It can be reasoned that if non-covalent binding units are properly introduced in the gelator molecules, well defined arrays of these units could be found in the fibres that percolate a gel. Therefore, these systems would contain host surfaces suited for the interaction of multivalent guest molecules with the adequate positioning of functional groups (see Scheme 1)

Departament de Química Inorgànica i Orgànica; Universitat Jaume I, 12071, Castelló, Spain.E-mail:miravet@uji.es; escuder@uji.es; Fax: +34 964728214; Tel: +34964729154
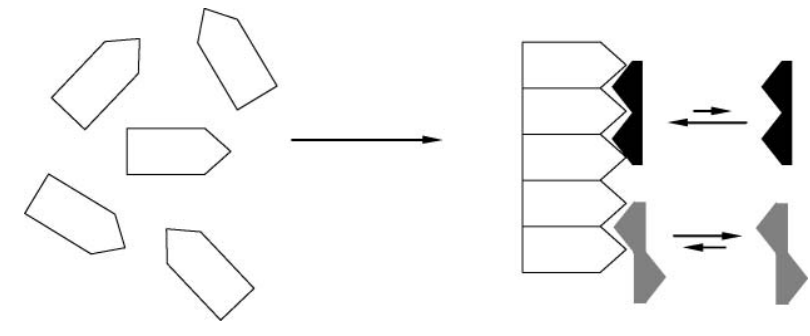

Scheme 1 Molecular recognition in the interaction of different substrates with a self-assembled fibre.

Only a few reports have been concerned so far with the selective interaction of molecules with the fibres of supramolecular gels. Shinkai and co-workers showed that a supramolecular organogel formed by a perylenediimide derivative was able to selectively signal the presence of 2,6-dihydroxynaphthalene by a change in color associated with charge-transfer interactions between the host molecule and the molecules in the gel fibres. ${ }^{8}$ Hamachi and co-workers reported the use of supramolecular gels in the molecular recognition of phosphates. ${ }^{9}$ Additionally, systems based on supramolecular gels have been used as a stationary phase for electrophoresis ${ }^{10}$ and for the separation of enantiomeric amino acids. $^{11}$

Here we report, as a proof of principle, the study of supramolecular gels as self-assembled multivalent hosts. For this purpose we have studied the interaction of phenol, 4-nitrophenol, different positional isomers of dihydroxybenzene and 2,7dihydroxynaphthalene with the supramolecular gels formed in acetonitrile by the low molecular weight gelators $\mathbf{1}$ and $\mathbf{2}$ (see Chart 1).

These and related molecules have been described by us as versatile gelators and used as templates for formation of inorganic materials, reactive gels or as supports for metallocatalysis. ${ }^{12}$ An aggregation model based on extended conformations and multiple $\mathrm{H}$-bonding interactions has been proposed for this family of gelators (see Scheme 2). Previous results such as the similar gelation properties of $\mathbf{1}, \mathbf{2}$ and an isomer of $\mathbf{2}$ derived from 

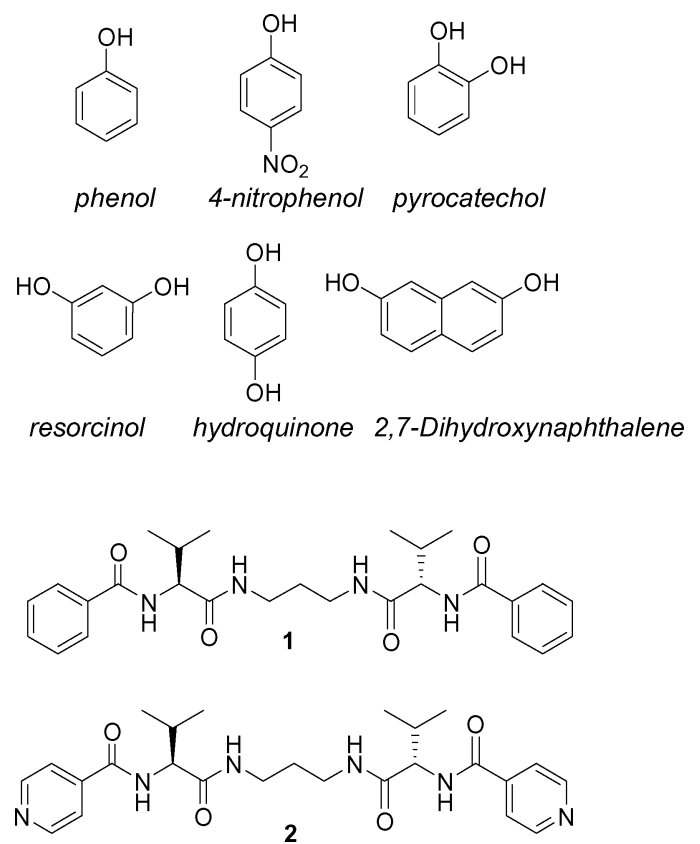

Chart 1 Structure of the gelators and phenolic substrates studied.

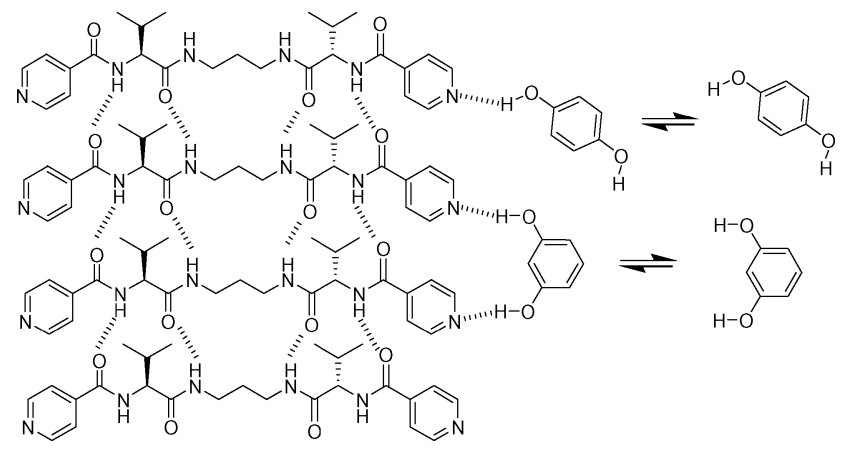

Scheme 2 Schematic model for the aggregation of $\mathbf{2}$ and its interaction with resorcinol and hydroquinone.

nicotinic acid indicated that the pyridine groups in $\mathbf{2}$ are not participating in the aggregation through hydrogen bonding. ${ }^{12 b, c}$ Additionally, gelator $\mathbf{2}$ has been shown to present free pyridine groups capable of interacting with palladium. ${ }^{12 \mathrm{c}}$ In the present study, it was envisaged that the presence of pyridine moieties in gelator 2 could be exploited advantageously for the interaction with phenol-type compounds through hydrogen bonding interactions as illustrated in Scheme 2.

\section{Results and discussion}

In order to asses the interaction of the different substrates with the studied gel networks, NMR relaxometry studies were carried out. We have shown previously that the measurement of NMR relaxation rates is a very convenient way to monitor the interaction of molecules with the gel fibres. ${ }^{13}$ The significant reduction of mobility associated with the adsorption on the fibre surface can result in a very notable reduction of the measured relaxation times. It has been shown that the formation of gels does not affect the relaxation time of molecules in solution that do not interact appreciably with the gel fibres despite the increase in macroscopic viscosity experienced upon gel formation. ${ }^{13,14}$ This fact is in agreement with a gel being formed by big pools of solvent entrapped in a network. ${ }^{15}$ In this work, we have measured the transversal relaxation time $\left(T_{2}\right)$ of the different substrates in the presence of the gels formed in acetonitrile by compound $\mathbf{2}$ and with the analogous gelator $\mathbf{1}$ in which the pyridine unit of $\mathbf{2}$ has been replaced by a phenyl moiety. The study of intermediate situations where aggregates are present but the gels are not formed was not possible due to the cooperative nature of self-assembly that gives places to a sharp change from free molecules to gels around a critical concentration value. ${ }^{16}$

The measured relaxation times obtained in the presence of the gel networks can be related to the relaxation time of the free molecules and that of the fibre-bound molecules as shown in eqn (1) (considering a fast exchange between adsorbed and free molecules in the NMR time scale). ${ }^{17}$ In eqn (1) $T_{\text {obs }}$ corresponds to the measured relaxation time of the studied substrate in the presence of the gel network, $T_{F}$ is the relaxation of the free substrate and $T_{B}$ the relaxation of the substrate bound to the surface of the gel network. Additionally, $x_{F}$ and $x_{B}$ represent, respectively, the molar fraction of free and bound substrate molecules.

$$
\frac{1}{T_{o b s}}=\frac{1}{T_{F}} x_{F}+\frac{1}{T_{B}} x_{B}
$$

A convenient way to compare the affinity of the different substrates for the surface of the fibres is to consider the value of the surface relaxivity, $\rho$ (see eqn (2) and (3)). This magnitude is analogous to that used, for example, in studies of porosity determination by NMR relaxometry. ${ }^{18}$ As found previously in NMR studies of porosity, our results indicate that the relaxation time of the fibre-bound substrate is much higher than that corresponding to the free species. ${ }^{18 b, c}$ Therefore, $1 / T_{\text {obs }}$ represents a good approximation to the value of $\rho$ and eqn (3) can be transformed to eqn (4).

$$
\begin{gathered}
\rho=\frac{1}{T_{B}} x_{B} \\
\rho=\frac{1}{T_{o b s}}-\frac{1}{T_{F}} x_{F} \\
\rho \cong \frac{1}{T_{o b s}}
\end{gathered}
$$

The value of $\rho$ is dependant on the strength of the interaction between the substrate and the gel network. Stronger interactions would produce a more intense reduction of $T_{B}$ values (due to restricted mobility of adsorbed molecules) and higher values of $x_{B}$ (due to increased molar fraction of adsorbed molecules). Consequently, it can be rationalized that the higher the measured $\rho$ values, the higher the affinity of a given substrate for the studied gel network.

The results obtained for the relaxivity measurements in samples containing different amounts of substrates and gelator are shown in Table 1. These samples were prepared by gelation in the presence of the phenolic additives. It has to be mentioned that similar results were obtained when the additives were diffused into the gels formed previously. It can be noticed that all the 
Table 1 Measured NMR (300 MHz) $T_{2}$ relaxation times $\left(T_{o b s}\right)$ and calculated surface relaxivity $(\rho)$ in $\mathrm{CD}_{3} \mathrm{CN}$ gels at $30^{\circ} \mathrm{C} .{ }^{a b c}$

\begin{tabular}{|c|c|c|c|c|}
\hline Substrate & Gelator & $T_{f} / \mathrm{s}^{b}$ & $T_{o b s} / \mathrm{s}$ & $\rho / \mathrm{s}^{-1}$ \\
\hline phenol & 1 & 6.3 & 1.25 & $0.73(0.11)$ \\
\hline 4-nitrophenol & 1 & 5.1 & 0.58 & $1.66(0.25)$ \\
\hline pyrocatechol & 1 & 6.6 & 0.57 & $1.68(0.21)$ \\
\hline resorcinol & 1 & 6.7 & 0.51 & $1.89(0.32)$ \\
\hline hydroquinone & 1 & 7.3 & 0.72 & $1.32(0.11)$ \\
\hline $\mathrm{DHN}^{c}$ & 1 & 5.9 & 0.39 & $2.56(0.35)$ \\
\hline phenol & 2 & 6.3 & 1.53 & $0.57(0.13)$ \\
\hline 4-nitrophenol & 2 & 5.1 & 0.61 & $1.56(0.19)$ \\
\hline pyrocatechol & 2 & 6.6 & 0.60 & $1.59(0.14)$ \\
\hline resorcinol & 2 & 6.7 & 0.16 & $6.17(0.87)$ \\
\hline hydroquinone & 2 & 7.3 & 0.71 & $1.34(0.12)$ \\
\hline $\mathrm{DHN}^{c}$ & 2 & 5.9 & 0.23 & $4.35(0.40)$ \\
\hline
\end{tabular}

${ }^{a}$ Gels formed in acetonitrile. Concentration of gelator: $21 \mathrm{mM}$. Concentration of substrates: $42 \mathrm{mM}$. Values in parentheses indicate the estimated error. ${ }^{1} \mathrm{H}$ NMR signals monitored (ppm): phenol, 7.23; 4nitrophenol, 8.12; pyrocatechol, 6.82; resorcinol, 7.03; hydroquinone 6.66; DHN, 7.65. ${ }^{b}$ Relaxation time of free substrates; calculated for a $21 \mathrm{mM}$ solution of the studied substrate in the absence of gelator. ${ }^{c} \mathrm{DHN}=2,7$ dihydroxynaphthalene.

studied molecules present analogous $T_{2}$ values $(5-7 \mathrm{~s})$ and that the relaxation times are considerably reduced in the presence of the gel networks formed both by $\mathbf{1}$ and $\mathbf{2}$. This indicates that an interaction with the self-assembled fibres is detected in all the cases. The significantly higher reduction of $T_{2}$ values experienced in the presence of gels by 4-nitrophenol ( $T_{\text {obs }}=0.58$ and $\left.0.61 \mathrm{~s}\right)$ or dihydroxy compounds $\left(T_{o b s}<0.7 \mathrm{~s}\right)$ as compared to phenol $\left(T_{o b s}=\right.$ 1.25 and $1.53 \mathrm{~s}$ ) should be noted. These data support the fact that hydrogen bonding can be an important intermolecular interaction for the adsorption of the substrates on the fibres. As mentioned above, the affinity of the different substrates for the fibres can be conveniently compared considering the values of $\rho$. In order to visualize better the set of values of $\rho$ shown in Table 1, they have been graphically represented in Fig. 1.

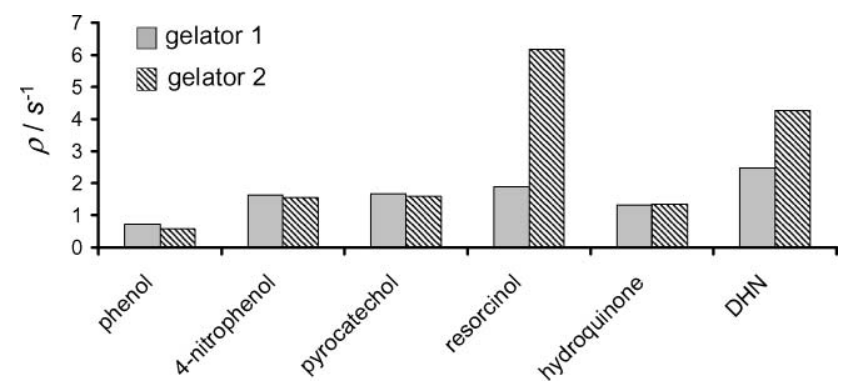

Fig. 1 Surface relaxivity $(\rho)$ in acetonitrile gels at $30^{\circ} \mathrm{C}(300 \mathrm{MHz}$ NMR, concentration of gelator: $21 \mathrm{mM}$; concentration of substrates: $42 \mathrm{mM}$; $\mathrm{DHN}=2,7$-dihydroxynaphthalene).

It can be noticed that for all the substrates except resorcinol and 2,7-dihydroxynaphthalene, very similar $\rho$ values were measured in the gels formed by $\mathbf{1}$ and $\mathbf{2}$. These results indicate that, for those substrates, the presence of pyridine $\mathrm{H}$-bond acceptor units in the gel fibres is not influencing noticeably their adsorption on the fibres. The reduced relaxation times measured in these cases, as compared to those obtained in the absence of gelator, could be ascribed to non-specific interactions with the polar moieties of the fibres. As is highlighted in Fig. 1, remarkably, a very significant
Table 2 Measured NMR (300 MHz) $T_{2}$ relaxation times in $\mathrm{CD}_{3} \mathrm{CN}$ gels of compound $\mathbf{2}$ with variable gelator/substrate ratios

\begin{tabular}{llcc}
\hline Substrate & {$[$ Gelator] $/ \mathrm{mM}$} & Substrate/gelator ratio & $T_{\text {obs }} / \mathrm{s}$ \\
\hline phenol & 21 & 2 & 1.53 \\
phenol & 21 & 10 & 1.69 \\
resorcinol & 21 & 2 & 0.16 \\
resorcinol & 21 & 10 & 0.17 \\
resorcinol & 10 & 0.5 & 0.22 \\
resorcinol & 10 & 4 & 0.27 \\
\hline
\end{tabular}

difference in the relaxivity is observed when the interaction of resorcinol with the gels formed by $\mathbf{1}$ and $\mathbf{2}$ is compared. In this case, the presence of pyridine moieties in the gel fibres results in more than a threefold increase in the measured relaxation rate that must be associated with a higher affinity of this substrate for the gel fibres. These results fit well with a two point binding model as proposed in Scheme 2 where the two hydroxy groups of the resorcinol bind the pyridine units of adjacent gelator molecules. It seems that the pyrocatechol and hydroquinone geometry preclude a two point interaction with the pyridine groups and, as a matter of fact, their surface relaxivity values in the presence of the gel formed by $\mathbf{2}$ are comparable to that of 4-nitrophenol.

As shown in Table 2, studies carried out for variable concentrations of substrate and gelator (in the range of experimentally feasible concentrations) showed little variation of $T_{2}$ values as compared to the accuracy of the measurements and precluded the calculation of binding constants. For example, it is shown in Table 2 that the value of $T_{2}$ for resorcinol in a gel formed by 2 showed only a decrease of $\mathrm{ca}$. $10 \%$ upon a fivefold increase of the concentration of this substrate.

X-Ray diffraction data of the xerogel formed by 2 (Fig. 2) revealed the microcrystalline nature of the aggregates with the presence of a low angle peak at $14.8 \AA$ that is in accordance with an extended conformation of $\mathbf{2}$ in the fibres. A peak is also found at $c a .4 .5 \AA$ that by analogy to previously reported xerogels, can be ascribed to the periodicity in the direction of the hydrogen bonding array. A tentative model for the fibre aggregates can be built with molecular mechanics (see Fig. 3) based on our previous findings for this family of molecules and on the data obtained from powder X-ray diffraction of the xerogel. The models obtained with molecular mechanics are in accordance with the spacing between pyridine groups detected by X-ray diffraction. As

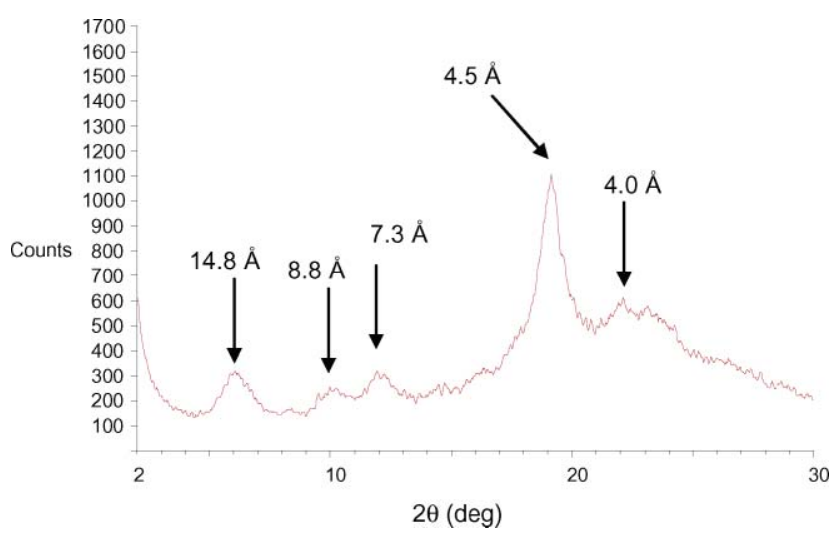

Fig. 2 X-Ray powder diffraction of a xerogel obtained from acetonitrile gel formed by compound 2 . 


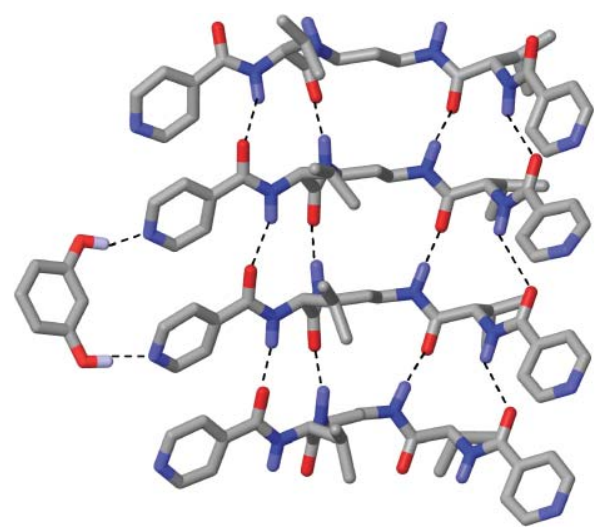

Fig. 3 Molecular model for the aggregation of gelator 2 and its interaction with resorcinol (AMBER* force-field, MACROMODEL 8.0).

shown in Fig. 3, the calculated structure for the interaction with resorcinol nicely agrees with a two point binding model of this molecule to the fibre aggregates. Additionally, the results shown for 2,7-dihydroxynaphthalene in Fig. 1 also indicate a selective interaction with the gel formed by the gelator $\mathbf{2}$ and support a divalent interaction model. In this case, the measured distance between the oxygen atoms in 2,7-dihydroxynaphthalene models is ca. $7.5 \AA$ which suggests that a two point interaction with nonadjacent pyridine units in the fibres could take place as shown in the model depicted in Fig. 4.

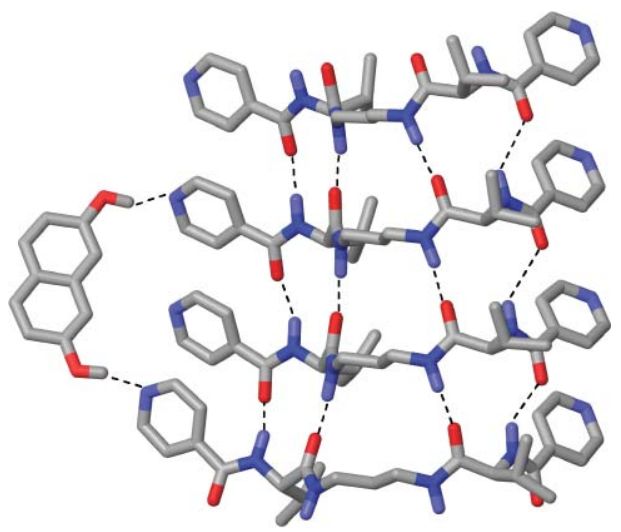

Fig. 4 Molecular model for the aggregation of gelator 2 and its interaction with 2,7-dihydroxynaphthalene (AMBER* force-field, MACROMODEL 8.0).

In order to obtain further experimental support to the described results, NMR experiments were carried out for equimolar mixtures of phenol, hydroquinone and resorcinol in the presence of the gels formed by $\mathbf{1}$ and $\mathbf{2}$. Very interestingly, the selective interaction of resorcinol with the gel formed by the pyridine derivative $\mathbf{2}$ permits the selective suppression of the signal of that molecule upon recording a $T_{2}$ filtered spectrum (see Fig. 5). In the case of the gel formed by $\mathbf{1}$, this was not possible due to the similar $T_{2}$ values of all the species.

Additionally, the macroscopic behaviour of the gels also showed marked differences in the presence of resorcinol. As can be seen in Fig. 6, gels of $\mathbf{2}$ that contain resorcinol showed reduced stability as compared to those containing the other phenolic additives. In Table 3 are shown the values of thermal stability measured

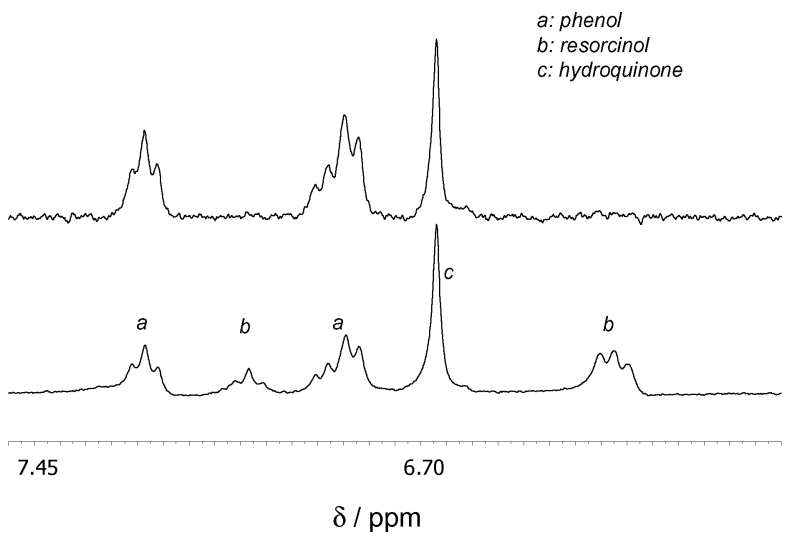

Fig. $5{ }^{1} \mathrm{H}$ NMR spectra of phenol, hydroquinone and resorcinol (20 mM each) in the gel formed by $2(10 \mathrm{mM}),\left(\mathrm{CD}_{3} \mathrm{CN}, 30{ }^{\circ} \mathrm{C}\right)$. Bottom: conventional ${ }^{1} \mathrm{H}$ spectrum; top: $T_{2}$ filtered ${ }^{1} \mathrm{H}$ spectrum (CPMG pulse sequence; total time for echoes $=3 \mathrm{~s}$ ).

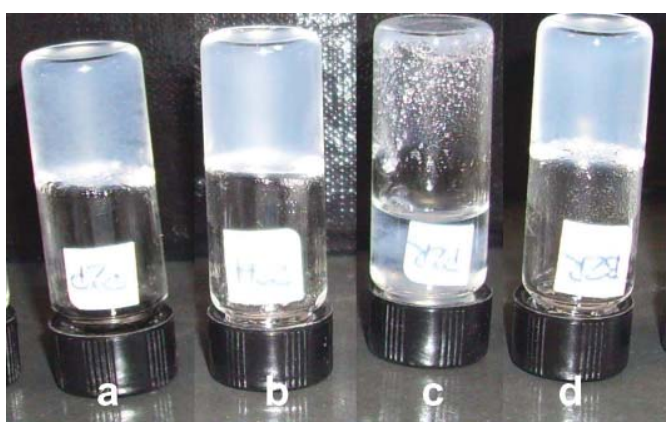

Fig. 6 Pictures of the samples prepared by gelation of acetonitrile in the presence of phenol derivatives. In all the samples the concentrations of gelator and phenol derivative were respectively $21 \mathrm{mM}$ and $50 \mathrm{mM}$. a) 2 and phenol; b) 2 and hydroquinone; c) $\mathbf{2}$ and resorcinol; d) $\mathbf{1}$ and resorcinol.

for the gels formed by $\mathbf{1}$ and $\mathbf{2}$ in the presence of different phenolic additives. It can be seen that the presence of resorcinol dramatically affects the thermal stability of the gels formed by the pyridine derived gelator 2 . The gels formed by $\mathbf{2}$ in the presence of a fivefold excess of hydroquinone or phenol present a similar thermal stability $\left(T_{g} \mathrm{ca} .70^{\circ} \mathrm{C}\right)$ while the corresponding gel with resorcinol is not self-sustained at room temperature. This behaviour could be tentatively ascribed to a weakening of the fibril packing or to the different solvation properties associated to the resorcinol decorated fibres. On the other hand, the properties of

Table 3 Thermal stability for the gels formed by $\mathbf{1}$ and $\mathbf{2}$ in the presence of phenolic additives ${ }^{a}$

\begin{tabular}{llr}
\hline Gelator & Additive & $T_{g} /{ }^{\circ} \mathrm{C}^{b}$ \\
\hline $\mathbf{2}$ & phenol & 70 \\
$\mathbf{2}$ & hydroquinone & 75 \\
$\mathbf{2}$ & resorcinol & $<25$ \\
$\mathbf{1}$ & phenol & 80 \\
$\mathbf{1}$ & hydroquinone & 85 \\
$\mathbf{1}$ & resorcinol & 80
\end{tabular}

${ }^{a}$ Concentration of gelator $21 \mathrm{mM}$. Concentration of additives $100 \mathrm{mM}$.

${ }^{b}$ Temperature at which the formed gels are not self-sustained upon vial inversion. 
the gels formed by 1 remain basically unaltered in the presence of resorcinol.

\section{Conclusions}

In conclusion, the results indicate that low molecular weight molecules containing functional moieties can afford selfassembled fibrillar networks that present ordered arrays of binding groups. In the case reported here, it is shown, as a proof of principle, that the assembly produces multivalent surfaces containing $\mathrm{H}$-bond acceptor pyridine units with a disposition that is complementary to that found in for the hydroxy groups of resorcinol and 2,7-dihydroxynaphthalene. This allows their recognition in the presence of other related molecules, including positional isomers. A direct application of the findings in the selective suppression of the NMR signals of resorcinol in the presence of related substrates has been shown. This procedure may find application in the NMR analysis of complex mixtures. Moreover, the selective recognition is expressed also at the macroscopic level, the thermal stability of the gels being markedly affected by the presence of resorcinol as compared to other phenolic derivatives. The results described reveal that self-assembled fibrillar networks can be used in molecular recognition or positioning of substrates with a variety of potential applications, for example, in the development of sensing or stimuli responsive devices. Future work will be devoted to the study of the interaction of biologically relevant analytes with different gels.

\section{Experimental}

\section{Synthesis}

The preparation and characterization of gelators $\mathbf{1}$ and $\mathbf{2}$ has been described previously. ${ }^{12 b, c}$

\section{Organogel samples for NMR}

The gelator (6-18 $\mathrm{mmol})$, substrate $(6-72 \mathrm{mM})$ and internal standard (12 mmol of dioxane or tetrachloroethane) were dissolved in methanol:dichloromethane 1:1 and transferred into a $5 \mathrm{~mm}$ NMR tube. The solvent was fully removed carefully under vacuum. To these samples $\mathrm{CD}_{3} \mathrm{CN}$ was added and the septum-capped NMR tube was deoxygenated by four freeze-pump-thaw cycles (using a needle connected to a vacuum source). Then the septumcapped sample was heated gently until complete solubilization of the mixture and the hot NMR tube was immersed first for 5 minutes in a thermostatic bath at $90{ }^{\circ} \mathrm{C}$ and then for 5 minutes at $30{ }^{\circ} \mathrm{C}$. Afterwards the gel formed in this way was left to rest at room temperature for at least 12 hours before NMR measurements.

\section{NMR measurements}

NMR spectra corresponding to the relaxation experiments were recorded in a Varian Mercury instrument $\left(300 \mathrm{MHz}\right.$ for $\left.{ }^{1} \mathrm{H}\right)$ at $30{ }^{\circ} \mathrm{C} . T_{2}$ measurements were performed using the Carr-PurcellMeiboom-Hill (CPMG) sequence with dephasing times of $0.4 \mathrm{~ms}$. A minimum of 14 points were acquired for each of the relaxation experiments and a good fit of the data to a monoexponential decay was obtained in all the reported data.

\section{Thermal stability of the gels}

The corresponding gels were prepared in vials $(\mathrm{d}=1 \mathrm{~cm})$ and their thermal stability was checked using a thermostatic bath by vial inversion at different temperatures $\left(5^{\circ} \mathrm{C}\right.$ intervals $)$.

\section{X-Ray powder diffraction}

Data collection was performed at room temperature on a Bruker D4 Endeavor X-ray powder diffractometer using $\mathrm{Cu}-\mathrm{K}_{\mathrm{a}}$ radiation. Samples of the powdered xerogels were placed on a sample holder and data were collected for $2 \theta$ values between $2^{\circ}$ and $50^{\circ}$ with a step size of $0.04^{\circ}$ and a time step of $8 \mathrm{~s}$.

\section{Acknowledgements}

Financial support from Ministerio de Educación y Ciencia of Spain (CTQ2006-14984) and Universitat Jaume I (P1 1B2007-11) is acknowledged.

\section{Notes and references}

1 (a) J.-M. Lehn, Supramolecular Chemistry, VCH, Weinheim, 1995; (b) K. Ariga and T. Kunitake, Supramolecular Chemistry-Fundamentals and Applications, Springer, 2006.

2 (a) T. Ishi-i, M. Crego-Calama, P. Timmerman, D. N. Reinhoudt and S. Shinkai, Angew. Chem. Int. Ed., 2002, 41, 1924-1929; (b) T. Ishi-i, M. A. Mateos-Timoneda, P. Timmerman, M. Crego-Calama, D. N. Reinhoudt and S. Shinkai, Angew. Chem. Int. Ed., 2003, 42, 2300-2305.

3 (a) C. Schmuck, Angew. Chem. Int. Ed., 2007, 46, 5830-5833; (b) S. M. Biros and J. Rebek, Jr., Chem. Soc. Rev., 2007, 36, 93-104; (c) M. Yoshizawa and M. Fujita, Pure Appl. Chem., 2005, 77, 1107-1112.

4 D. M. Vriezema, M. Comellas, J. A. A. W. Elemans, J. J. L. M. Cornelissen, A. E. Rowan and R. J. M. Nolte, Chem. Rev., 2005, 105, $1445-1489$.

5 (a) A. Mulder, T. Auletta, A. Sartori, S. Del Ciotto, A. Casnati, R. Ungaro, J. Huskens and D. N. Reinhoudt, J. Am. Chem. Soc., 2004, 126, 6627-6636; (b) J. Huskens, A. Mulder, T. Auletta, C. A. Nijhuis, M. J. W. Ludden and D. N. Reinhoudt, J. Am. Chem. Soc., 2004, 126, 6784-6797; (c) M. J. W. Ludden, X. Y. Ling, T. Gang, W. P. Bula, H. J. G. E. Gardeniers, D. N. Reinhoudt and J. Huskens, Chem. Eur. J., 2008, 14, 136-142.

6 (a) M. Mammen, S.-K. Choi and G. M. Whitesides; Angew. Chem. Int. Ed., 1998, 37, 2755-2794; (b) A. Mulder, J. Huskens and D. N. Reinhoudt, Org. Biomol. Chem., 2004, 2, 3409-3424 and references therein.

7 (a) R. G. Weiss, P. Terech, (eds.), Molecular Gels, Materials with SelfAssembled Fibrillar Networks, Springer, 2005; (b) F. Fages, (ed.), Low Molecular Mass Gelators, Springer, 2005; (c) P. Terech and R. G. Weiss, Chem. Rev., 1997, 97, 3133-3159; (d) D. J. Abdallah and R. G. Weiss, Adv. Mater., 2000, 12, 1237-1247; (e) J. van Esch and B. L. Feringa, Angew. Chem. Int. Ed., 2000, 39, 2263-2354; $(f)$ O. Gronwald and S. Shinkai, Chem. Eur. J., 2001, 7, 4328-4334; $(g)$ L. A. Estroff and A. D. Hamilton, Chem. Rev., 2004, 104, 1201-1207; $(h)$ A. R. Hirst and D. K. Smith, Chem. Eur. J., 2005, 11, 5496-5508; (i) N. M. Sangeetha and U. Maitra, Chem. Soc. Rev., 2005, 34, 821-836.

8 P. Mukhopadhyay, Y. Iwashita, M. Shirakawa, S.-I. Kawano, N. Fujita and S. Shinkai, Angew. Chem. Int. Ed., 2006, 45, 1592-1595.

9 S. Yamaguchi, I. Yoshimura, T. Kohira, S. Tamaru and I. Hamachi, J. Am. Chem. Soc., 2005, 127, 11835-11841.

10 S. Mizrahi, J. Gun, Z. G. Kipervaser and O. Lev, Anal. Chem., 2004, 76, 5399-5404.

11 X. J. Fu, Y. Yang, N. X. Wang, H. Wang and Y. J. Yang, J. Mol. Recognit., 2007, 20, 238-244.

12 (a) J. F. Miravet and B. Escuder, Org. Lett., 2005, 7, 4791-4794; (b) B. Escuder, S. Marti and J. F. Miravet, Langmuir, 2005, 21, 6776-6787; (c) J. F. Miravet and B. Escuder, Chem. Commun., 2005, 5796-5798; (d) G. Roy, J. F. Miravet, B. Escuder, C. Sanchez and M. Llusar, J. Mater. Chem., 2006, 1817-1824. 
13 B. Escuder, M. Llusar and J. F. Miravet, J. Org. Chem., 2006, 71, 77477752.

14 D. C. Duncan and D. G. Whitten, Langmuir, 2000, 16, 6445-6452.

15 A. Masuda, K. Ushida, H. Koshino, K. Yamashita and T. Kluge, J. Am. Chem. Soc., 2001, 123, 11468-11469.

16 A. R. Hirst, I. A. Coates, T. R. Boucheteau, J. F. Miravet, B. Escuder, V. Castelletto, I. W. Hamley and D. K. Smith, J. Am. Chem. Soc., 2008, 130, 9113-9121.
17 (a) H. J. Schneider and A. Yatsimirsky, Principles and methods in Supramolecular Chemistry, Wiley, 2000; (b) C. A. Schalley, (ed.) Analytical Methods in Supramolecular Chemistry, Wiley-VCH, 2006.

18 (a) A. T. Watson and C. T. P. Chang, Prog. Nucl. Magn. Reson. Spectrosc., 1997, 31, 343-386; (b) J. Uh and A. T. Watson, Ind. Eng. Chem. Res., 2004, 43, 3026-3032; (c) J. H. P. Colllins, L. F. Gladden, I. J. Hardy and M. D. Mantle, Appl. Magn. Reson., 2007, 32, 185204. 